\title{
Embodied computation in soft gripper
}

\author{
Stig Anton Nielsen \\ Dept. of Architecture \\ Chalmers Technical University \\ stig.nielsen@chalmers.se
}

\author{
Alexandru Dancu \\ Dept. of Applied IT \\ Chalmers Technical University \\ alexandru.dancu@gmail.com
}

\begin{abstract}
We designed, created and tested an underactuated soft gripper able to hold everyday objects of various shapes and sizes without using complex hardware or control algorithms, but rather by combining sheets of flexible plastic materials and a single servo motor. Starting with a prototype where simple actuation performs complex varied gripping operations solely through the material system and its inherent physical computation, the paper discusses how embodied computation might exist in a material aggregate by tuning and balancing its morphology and material properties.
\end{abstract}

\section{Categories and Subject Descriptors}

I.2.9 [Computing Methodologies]: Artificial IntelligenceRobotics

\section{INTRODUCTION}

This paper argues that human-robot interaction can simplify control systems, mechanisms and problems like collision detection by employing embodied computation. MacLennan describes embodied computation as "information processing in which the physical realization and the physical environment play an unavoidable and essential role" [4]. In this paper we will discuss embodied computation and suggest a material system that has reduced actuation complexity and performs gripping instead through an embodied material computation. Human-robot Interaction can manifest indirectly, in the sense that robots should be able to interact with the same environments humans do. This requires a certain resemblance between robots and humans: in behavior, morphology, materiality, and scale. But how do we determine what similarities are relevant, and should we mimic or replicate these mechanisms? What aspects of embodied computation are relevant to the design of material systems, morphology, and material behavior?

One major challenge in robotics is picking up and holding everyday objects without crushing them. For that we have created an adaptive, robust gripper able to interact

Permission to make digital or hard copies of part or all of this work for personal or classroom use is granted without fee provided that copies are not made or distributed for profit or commercial advantage and that copies bear this notice and the full citation on the first page. Copyrights for third-party components of this work must be honored. For all other uses, contact the Owner/Author.

Copyright is held by the owner/author(s).

HRI'14, Mar 03-06 2014, Bielefeld, Germany

ACM 978-1-4503-2658-2/14/03.

http://dx.doi.org/10.1145/2559636.2563691 with a large number of real objects from an office environment and with humans. Traditionally in computer-science, software has been developed and analyzed separately from hardware. In embodied computing the computation is seen as happening "as a physical system in continuing interaction with other physical systems (its environment)". [4]. Information processing is implicit here because the physical environment performs some computations "for free". Redström argues that computers can be seen as a kind of material, and that their computational capabilities must be combined with other kinds of materials in order to create a computational composite, so that the computer becomes "useful in design" [5]. This paper contributes a design of an underactuated gripper, a computational aggregate made up of material composites in a soft mechanical system, with an emphasis on morphology and material behavior interacting with the real environment.

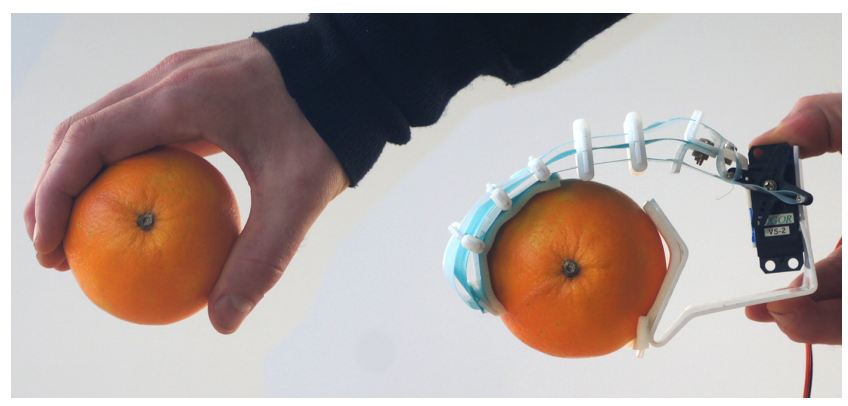

Figure 1: Gripper-hand for scale and comparison

\section{RELATED WORK}

Many robotic hand designs focus on mechanically replicating the human hand, controlling each joint independently using many actuators. On the other hand, underaction designs employ less actuators in order to control a larger number of joints. One of the first examples of an underactuated soft gripper, similar to a bicycle chain, was developed using pulleys and twenty articulations. It was able to conform to objects of arbitrary shapes [3]. However, the design of this gripper only permitted holding an object in one plane. Another example of a soft "universal" gripper could conform around a complex object from all sides, and hold it by contracting the granular material it was made from [1]. This is a good example of embodied computing where a computational composite is used. The granular material automatically computes and shapes around an object, simplifying and avoiding the problems multifingered robotic hands ex- 
perience when needing to compute the force and position required to control each finger. A simple design employing material intelligence can thus avoid both hardware and software complexities.

\section{DESIGN AND IMPLEMENTATION}

A simple gripper design employing this kind of material intelligence is composed of three main parts, a dynamic actuated arm, a servo, and a thumb. The thumb is an acrylic plastic plate, that bends into shape, with an added a layer of high friction material. It is positioned below / inside the dynamic arm so that it creates a static counterpart for the dynamic arm. This piece could be compared to the thumb or the palm of a hand. In our design, the dynamic, actuated arm consists of a central flexible core and upper and lower tendons. The tendons are attached to the tip of the core and run through plastic fittings fixed to the central core, allowing the tendons free linear movement (Fig. 1). "The tendons are actuated by a servo motor controlled by a looping prototype program that turns them clockwise, holds them, and then turns them counter-clockwise.

The flexible core is bendable, but static in length. When a tendon is actuated in a linear movement through the fittings, the central core will bend, firstly at the base, as well as the tip, resulting in a grasping movement. When parts of the arm are obstructed by either the object or the table, the gripper starts to bend in other parts along its length, conforming around the object. Due to the flexibility and softness of the materials, the gripper never crushes objects. On the contrary, the morphology and mechanism makes the grip firmer only as the weight of the objects increases. Force is applied incrementally, building up through the material, giving it a shape and conforming to it, resulting in a uniform tension around the object that becomes the final grip. Furthermore the tendon, being very close to the object, contributes the force of the grip.

Most daily objects are designed at human scale, therefore the scale of the gripper and thumb was constructed to match that of a human hand (see Fig. 1) enabling it to grasp most objects from an office environment. Deimel argues that constraints present in the environment must be exploited for robust grasping [2]. The inside of the thumb and the tip of the arm are coated with high friction material (foam), permitting it to hold objects of more than six times its own weight. The gripper all inclusive weighs $95 \mathrm{~g}$, and the stapler pictured 606g (Fig. 2, lower left).

\section{DISCUSSION}

We arrived to this design empirically by varying the length of the central core and tendons, the thickness of the central core, as well as changing the position for the plastic fittings. We found out that for this type of plastic for the tendons and central core, the optimal length is $15 \mathrm{~cm}$ (full length of a hand is $14-18 \mathrm{~cm}$ ). Shorter lengths of the central core and tendons limits the size of graspable objects, while longer sizes decreases the applied gripping force and introduces flexing to the left or right of the central gripper axis.

The position of the plastic fittings determine how the gripper bends. Fig. 2 shows different adaptations of the tendons and central core. The tension spreads through the material automatically computing the force and morphology necessary to grasp and hold the particular object. Design improvements can be done, and the balance between items in the material system seems to be of great importance, which is why it is prudent to make a modifiable design, so the balance can be tuned without having to build entire new prototypes. This notion might open an entire discussion of a second order actuation which could control and tune the balance of the material system e.g. moving the fittings or changing the stiffness of the core along the central core depending on the instance of a gripping action.

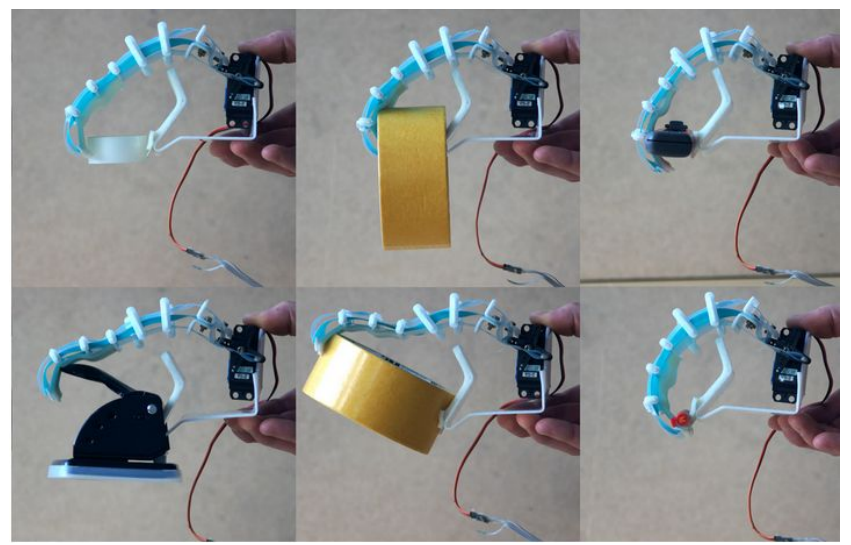

Figure 2: The material system adapts to the shape of each subject. Even small subjects are gripped by the outer tip and picked up. Heavy objects are squeezed in the gripper.

\section{CONCLUSION}

A soft gripper was designed, created, and tested by gripping objects from an office environment. This prototype showed that the force and the grasping method can be computed by the material, optimizing its morphology, exemplifying embodied computation in an underactuated soft gripper. When designing for increased embodied computation in soft robotics, some aspects of flexibility might decrease, but a surprisingly high robustness was achieved. When assembled, the gripper only fails on single case grasping tasks, it never fails mechanically or breaks itself due to incorrect usage. It seems relevant to let material properties compute active aspects like pressure and collision. The morphology and scale seems relevant to mimic directly, in this case when dealing with everyday objects already designed for the scale and morphology of the hand.

\section{REFERENCES}

[1] Brown, E., Rodenberg, N., Amend, J., Mozeika, A., Steltz, E., Zakin, M. R., Lipson, H., and Jaeger, H. M. Universal robotic gripper based on the jamming of granular material. Proceedings of the National Academy of Sciences 10\%, 44 (2010), 18809-18814.

[2] Deimel, R., Eppner, C., Alvarez-Ruiz, J., Maertens, M., and Brock, O. Exploitation of environmental constraints in human and robotic grasping.

[3] Hirose, S., and Umetani, Y. The development of soft gripper for the versatile robot hand. Mechanism and machine theory 13, 3 (1978), 351-359.

[4] MacLennan, B. J. Aspects of embodied computing. Tech. rep., Technical Report UTCS-08-610, University of Tennessee, 2008.

[5] Vallgårda, A., and Redström, J. Computational composites. In Proceedings of the SIGCHI conference on Human factors in computing systems, ACM (2007), 513-522. 